\title{
Influence of Pore Size on Fracture Strength of Porous Ceramics*
}

\author{
Keigo YOSHIDA $^{* *}$, Hironori TSUKIDATE ${ }^{* * *}$, Akira MURAKAMI ${ }^{* * * *}$ \\ and Hiroshi MIYATA ${ }^{* * * *}$ \\ ${ }^{* *}$ Automotive Systems, Hitachi, Ltd, \\ 4-6-1 Midorigaoka, Atsugi, Kanagawa, Japan \\ ${ }^{* * *}$ Power Systems, Hitachi, Ltd, \\ 3-1-1 Saiwai-cho, Hitachi, Ibaraki, Japan \\ ****Graduate School of Science and Technology, Hirosaki University, \\ 3 Bunkyo-cho, Hirosaki, Aomori, Japan \\ E-mail: miyata@cc.hirosaki-u.ac.jp
}

\begin{abstract}
Porous ceramics possess the excellent penetration and adiabatic characteristics, etc., and are used as heatproof filter materials for environmental equipments, etc. Moreover, porous ceramics controlled with porosity and pore size in the wide range have been actively developed. However, how the strength characteristics of porous ceramics are influenced by porosity and pore size of the material are not understood still enough. In this research, the evaluation tests on fracture strength, fracture energy and fracture toughness of porous alumina ceramics which porosity are almost equal, while pore sizes are different mutually were performed, and the relation between the pore size and the fracture strength was studied. The tests results show that the dispersion of fracture strength data is few though fracture strength of porous ceramics is lower than that of high-density ceramics. The relation based on linear fracture mechanics between the defect size and the fracture strength is valid when the one that a pore accompanies with the peculiar defect of the material was regarded as a defect size. In addition, fracture energy increases with the increase of pore size, and this seems based on a crooked propagation path of a crack. Finally, the process zone fracture model with considering the effect of the pore and grain size of the material is proposed. According to this model, for all pore size and crack length, it was shown that the fracture strengths of cracked specimens are evaluated.
\end{abstract}

Key words: Porous Ceramics, Material Testing, Bending, Fracture Strength, Pore Size, Strength Evaluation, Fracture Toughness

\section{Introduction}

Porous ceramics possess the excellent penetration and adiabatic characteristics besides the heat resistant and the corrosion resistant characteristics and they are utilized as heatproof filter materials for environmental equipments etc ${ }^{(1)}$. And, porous ceramics controlled with porosity and pore size for the wide range have been still actively developed. Although porous ceramics are commonly used under the environmental conditions where the fracture strength properties of the material are not so important, hereafter it is expected that they will be used under the conditions where the reliability of the fracture strength properties are required. Thus, establishment of the criterion about the strength design of porous ceramics is desired. However, how the strength characteristics of porous ceramics are influenced by various parameters such as pore size, pore shape and porosity is not 
understood still enough.

An experimental formula expressing an exponential decrease of the fracture strength of porous ceramics along with increase of the porosity has been presented ${ }^{(2)}$. It is understood that the fracture strength is influenced by the difference of porosity associated with the net cross-sectional area. Considering the fact that the fracture strength of high-density ceramics is dominated by the defect size, it is deduced that pores dispersed in porous ceramics behave like defects. Thus, how the pore size affects the fracture strength of the material should be discussed. It has been suggested that the fracture strength properties of porous materials that have closed pores are superior to those of the materials that have open pores. It has been also suggested that plural pores adjacent each other behave like the large defect that decreases the fracture strength of the material ${ }^{(3)}$. Furthermore, in the study about the fracture toughness, that is important to the strength evaluation of brittle materials, it has been clarified that the fracture toughness for relatively large crack shows almost constant, but that value for small cracks shows relatively low ${ }^{(4)-(6)}$. It is thought that pores behave like a crack.

In this study, in order to establish the criterion about the strength design of porous ceramics, the bending tests were carried out about porous ceramic specimens that have almost equal porosity but significantly different pore size. And the fracture strength characteristics associated with the pore size were evaluated.

\section{Experimental Procedures}

\subsection{Porous Ceramic Specimens}

Specifications of porous ceramic samples are shown in Table 1. Ten alumina-based ceramic samples (accessory ingredients are Silica, Titania and so on.) fabricated by KYOCERA Corporation were tested. The porosity of these samples are almost equal to each other, while the pore sizes are significantly different. Four-point bending tests were carried out on plain specimen, SENB (Single Edge Notched Beam) specimen and CN (Chevron Notched) specimen. Since the samples have large pores, the size of the plain specimen is to be $8 \times 6 \times 70 \mathrm{~mm}^{3}$ which are two times larger than those defined in the JIS R 1601. Each fifteen plain specimens are tested for the samples \#1 \#10 shown in Table 1. Each ten SENB specimens with the size of $6 \times 8 \times 70 \mathrm{~mm}^{3}$ were tested for the samples \#1, \#2, \#3, \#5 and \#6 to evaluate the fracture toughness. Notches of $0.1 \sim 3.0 \mathrm{~mm}$ in depth and $0.3 \mathrm{~mm}$ in width were artificially introduced individually in the height $(8 \mathrm{~mm})$ direction of the center in the specimen length. The sizes of the $\mathrm{CN}$ specimen used for stable fracture test are $9 \times 12 \mathrm{x}$ $110 \mathrm{~mm}^{3}$. Chevron notch was artificially introduced in the center of the longitudinal direction as shown in Fig.1, and was tested of the samples \#1, \#2, \#3, \#5 and \#6.

Table 1 Specifications of porous ceramics tested.

\begin{tabular}{|c||c|c|c|}
\hline No. & $\begin{array}{c}\text { Porosity } \\
\%\end{array}$ & $\begin{array}{c}\text { Pore size (Ave.) } \\
\mu \mathrm{m}\end{array}$ & $\begin{array}{c}\text { Crystal size } \\
\mu \mathrm{m}\end{array}$ \\
\hline \hline$\# 1$ & 39 & 6 & $13-15$ \\
\hline$\# 2$ & 40 & 18 & $38-43$ \\
\hline$\# 3$ & 38 & 30 & $63-105$ \\
\hline$\# 4$ & 37 & 40 & $88-125$ \\
\hline$\# 5$ & 38 & 50 & $105-149$ \\
\hline$\# 6$ & 37 & 85 & $149-210$ \\
\hline$\# 7$ & 35 & 120 & $210-297$ \\
\hline$\# 8$ & 35 & 220 & $297-420$ \\
\hline$\# 9$ & 32 & 340 & $420-590$ \\
\hline$\# 10$ & 34 & 490 & $590-840$ \\
\hline
\end{tabular}



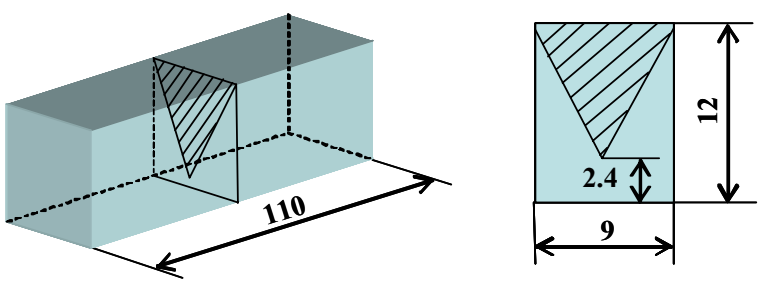

Fig.1 Configuration of a Chevron notched specimen.

\subsection{Testing Methods}

Four-point bending load was applied by means of the INSTRON 4464 testing machine (max. load $2 \mathrm{kN}$ ). In the bending tests of the plain and SENB specimens, the outer and inner fulcrum spans and the crosshead speed are $60 \mathrm{~mm}, 20 \mathrm{~mm}$ and $0.05 \mathrm{~mm} / \mathrm{min}$ respectively. And those for the $\mathrm{CN}$ specimen are $90 \mathrm{~mm}, 30 \mathrm{~mm}$ and $0.01 \mathrm{~mm} / \mathrm{min}$ respectively.

The maximum stress $\sigma$ induced with bending load of the plain specimen is calculated by the following equation.

$$
\sigma=\frac{3 P\left(L_{1}-L_{2}\right)}{2 w t^{2}}
$$

Where, $P$ is the applied load, $w$ and $t$ are the width and the height of specimen tested, $L_{1}$ and $L_{2}$ are the outer and the inner fulcrum spans, respectively.

The stress intensity factor $K_{\mathrm{I}}$ of the SENB specimen is calculated by the following equation.

$$
\begin{aligned}
K_{\mathrm{I}} & =F \sigma \sqrt{\pi a} \\
F & =1.99-2.47 \beta+12.97 \beta^{2}-23.17 \beta^{3}+24.80 \beta^{4} \\
\beta & =a / t
\end{aligned}
$$

Where, $\sigma$ is the fracture stress, $a$ is the notch depth and $F$ is the shape factor.

The fracture energy $\Gamma$ of the $\mathrm{CN}$ specimen is calculated by the area $U$ surrounded by the load-displacement curve and the displacement axis, and the area $A$ of the fracture surface of the specimen as follows.

$\Gamma=\frac{U}{2 A}$

Morphology of the fractured surface was observed through a CCD camera, and microscopic observation on the surface was also performed by SEM (HITACHI S-800, accelerating voltage $25 \mathrm{kV}$ ) after the Ag sputtering.

\section{Results and Discussion}

\subsection{Bending Fracture Strength of Plain Specimen}

Weibull statistics of the four-point bending fracture strengths are shown in Fig.2. Weibull modulus are 17.2 26.4, which are larger than those of high-density ceramics reported ${ }^{(7)}$. It is known that the fracture strength of high-density ceramics is influenced by dominant defects that exist sparsely in the material. In the porous ceramics, however, many pores that will be fracture origin exist randomly throughout the material, and then the scattering width of the fracture strength becomes narrow. On the other hand, relation between the fracture strength $\sigma_{\mathrm{c}}$ and the pore size $d$ is shown in Fig.3. The average fracture strength decreases from 63 to 20MPa with increase of the pore size from 6 to $490 \mu \mathrm{m}$. Since the porosities are almost equal among the samples tested in this study, it is suggested that the effect of pore size should be considered in the fracture strength evaluation of the porous ceramics.

In the SEM observations on the fracture surfaces, some coarse defects, which are distinctly larger than the pore, were observed for the samples with a relatively small pore as shown in Fig.4. The size of the defects is about $50-140 \mu \mathrm{m}$. On the other hand, such a defect 
was not observed in the samples that have a pore size larger than $50 \mu \mathrm{m}$. It is thought that such a defect is formed due to coupling of pores in the production process and peculiar to each sample.

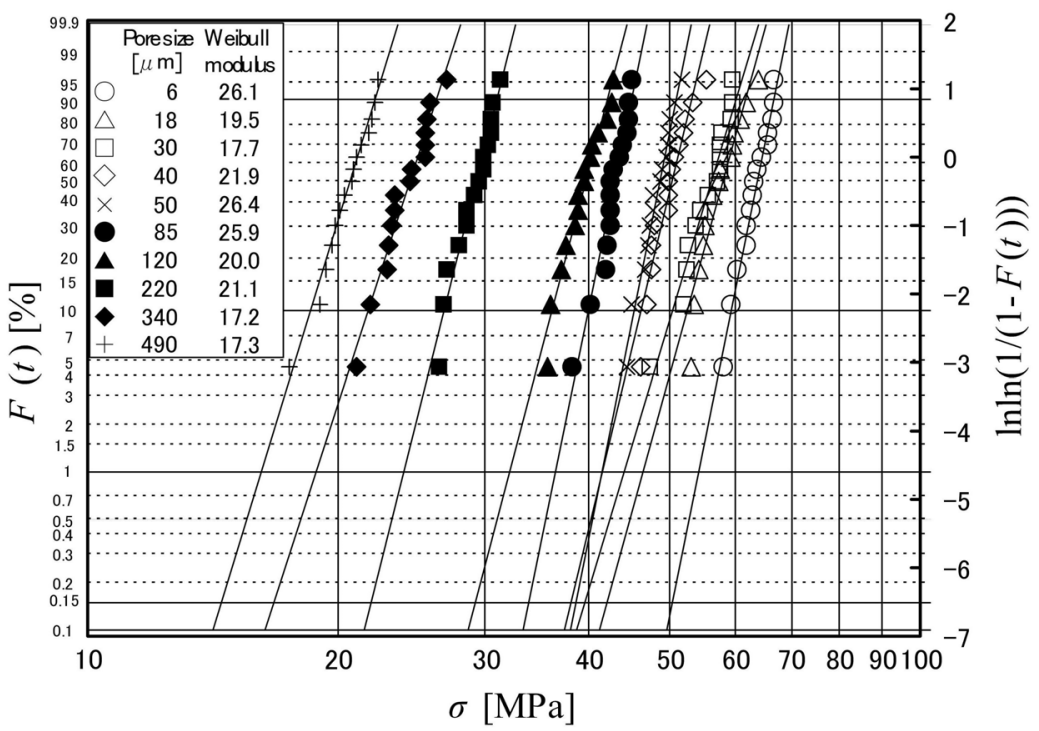

Fig.2 Weibull statistics of four-point bending fracture strength.

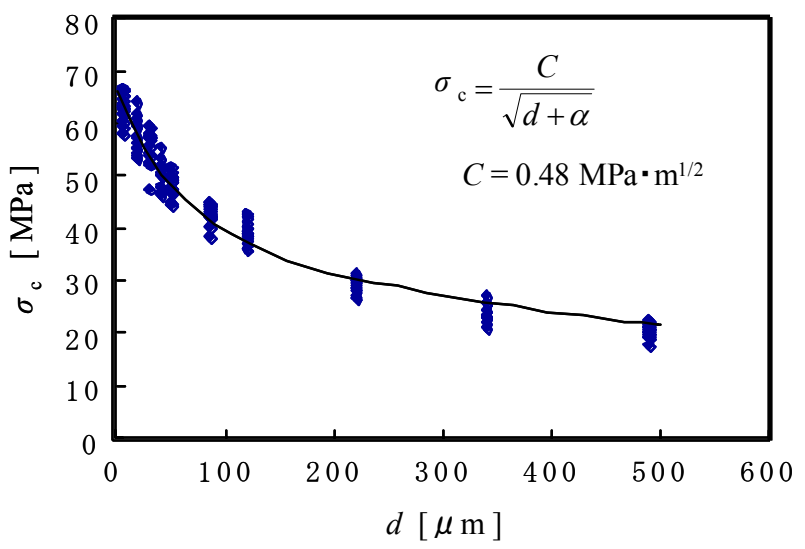

Fig.3 Relationship between fracture strength and pore size of the porous ceramics.

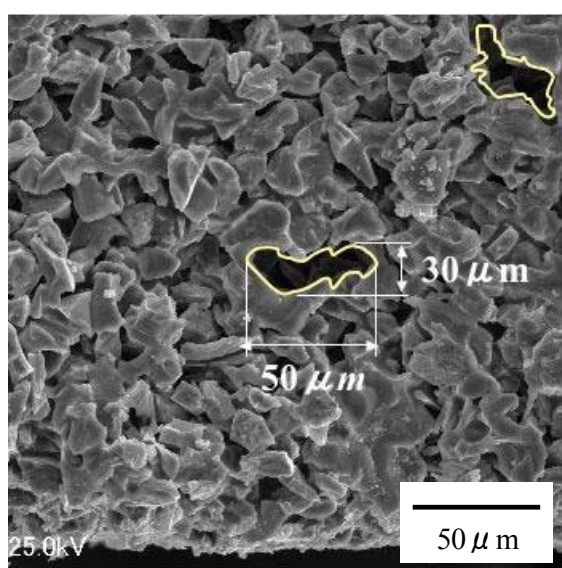

Fig.4 SEM observation of the fracture surface of plain specimen \#1 (pore size: $6 \mu \mathrm{m})$. 
In the macroscopic observations on the fracture surfaces, unevenness was observed, however, the fracture surface is relatively flat and many cleavage planes with grain size were observed in the microscopic scale. It is deduced that the fracture originates from the glass layer formed by segregation of impurities in the grain boundary region. It is assumed that the microscopic fracture occurs in the region between the peculiar defect and the adjacent pore, and successive fracture occurs along the grain boundary. The conditions for occurrence of the successive fracture determine the fracture strength of the material. It is assumed that the defect size $2(d+\alpha)$, which means the sum of the peculiar defect size $2 \alpha$ and the pore size $d$, dominates the fracture of material and the conditions for occurrence of the successive fracture are expressed by following equation.

$$
\sigma_{\mathrm{c}}=\frac{C}{\sqrt{d+\alpha}}
$$

The average defect size $\alpha$ is regarded to be about $50 \mu \mathrm{m}$ on the basis of the observations. A constant $C$ is determined to be $0.48 \mathrm{MPam}^{1 / 2}$ for fitting to the data. Since the above equation shows good agreement with correspondence to the experimental results, the fracture strength can be expressed by this equation regardless of the pore size. This result shows that the fracture strength of porous ceramics depends on the defect size on the basis of a constant intrinsic fracture toughness criterion. Such a dependency is similar to the fracture strength behavior of high-density ceramics.

\subsection{Fracture Toughness Test}

The fracture toughness value $K_{\mathrm{I}}$ obtained by the fracture strength of the SENB specimens through equation (2) arranged against the pore size $d$ was shown in Fig.5. The fracture toughness value of the sample \#1 (average pore size $6 \mu \mathrm{m}$ ) is slightly lower than those of the other samples. Scattering width of the fracture toughness data of the samples \#1 and \#2 $(18 \mu \mathrm{m})$ is narrow in comparison with those of the samples \#3, \#5 and \#6 (pore size are 30,50 and $85 \mu \mathrm{m}$, respectively). It is deduced that one of the reasons for it is the small scatter of the crystal grain size of the samples \#1 and \#2.

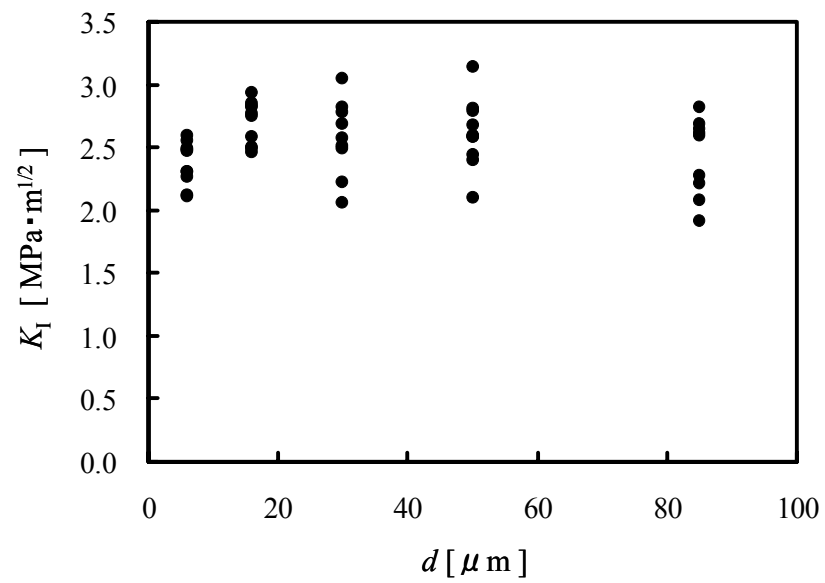

Fig.5 Dependence of the apparent fracture toughness on pore size.

The relations between the fracture toughness value $K_{\mathrm{I}}$ and the notch depth $a$ are shown in Fig.6. The fracture toughness value evaluated by the specimen with a small notch depth is lower than that with a relatively large notch depth and the latter is almost constant. It is known that the apparent fracture toughness value of high-density ceramics decreases with decrease of the crack length due to the saturation of the fracture strength ${ }^{(7)}$. It is considered, in case of small cracks, that the fracture strength is influenced by crystal grain. Similar to this, it is considered that the micro-structures associated with pores and crystal grains at the 
crack tip dominate the fracture strength of porous ceramics in the case where a crack depth is small. Although the fracture toughness tests of the specimen with a fatigue pre-crack were also carried out, there is no distinct difference in the fracture toughness values between the specimens with an artificial notch and a fatigue pre-crack.

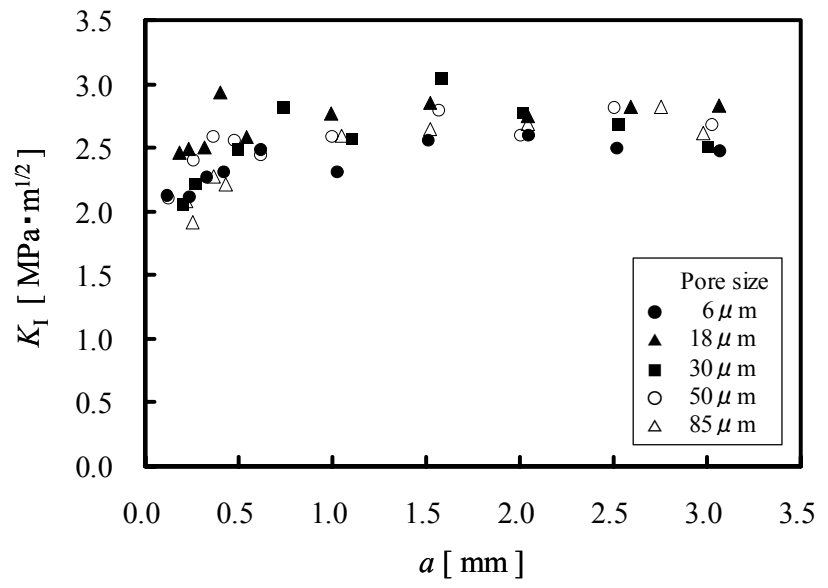

Fig.6 Effect of crack length on the apparent fracture toughness.

\subsection{Stable Fracture Test}

Load-displacement curves given by stable fracture tests are shown in Fig.7. Different from unstable fracture behavior, the load decreases gradually with increase of the displacement after applied load had reached maximum value. The maximum load applied to the $\mathrm{CN}$ specimen increases with increase of the pore size. This is different from the behavior observed between the fracture strength and the pore size for the plain specimen.

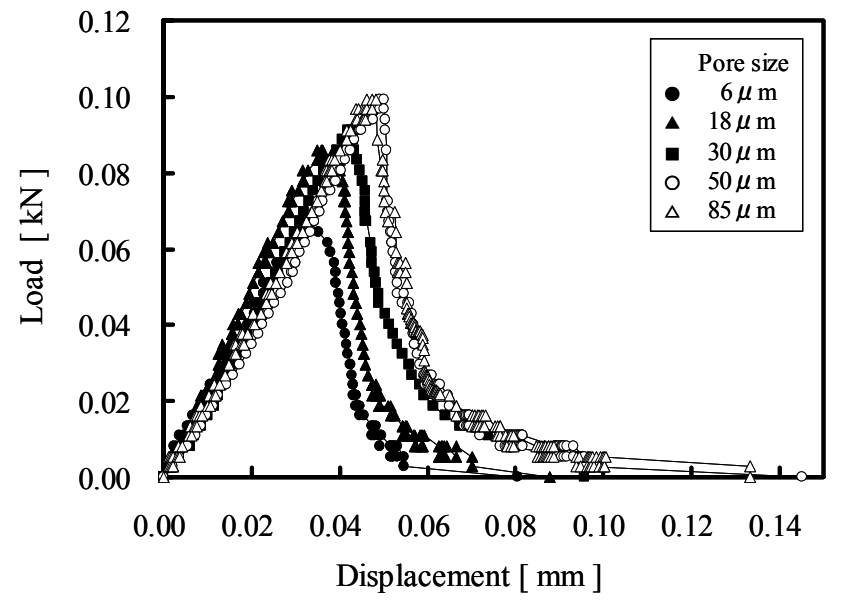

Fig.7 Load-displacement curves during fracture testing of Chevron notched specimens.

The relation between the fracture energy $\Gamma$ and the pore size $d$ is shown in Fig.8. The fracture energy of the porous ceramics in this study is higher than those of the high-density ceramics and increases from 41 to $89 \mathrm{~J} / \mathrm{m}^{2}$ with increase of the pore size from 6 to $85 \mu \mathrm{m}$. Although the fracture strength is decreased by pores, the fracture energy is increased by existing pores. This seems based on a crooked crack propagation path of a crack. The fact that the fracture energy increases with increase of the pore size proves that the crack propagation behavior is influenced by the pore size. Unevenness observed on the fracture surfaces of the specimens with a large pore size is larger than that with a small pore size as shown in Fig.9. This fact suggested that the crack propagation path is significantly deflected 
with a large pore.

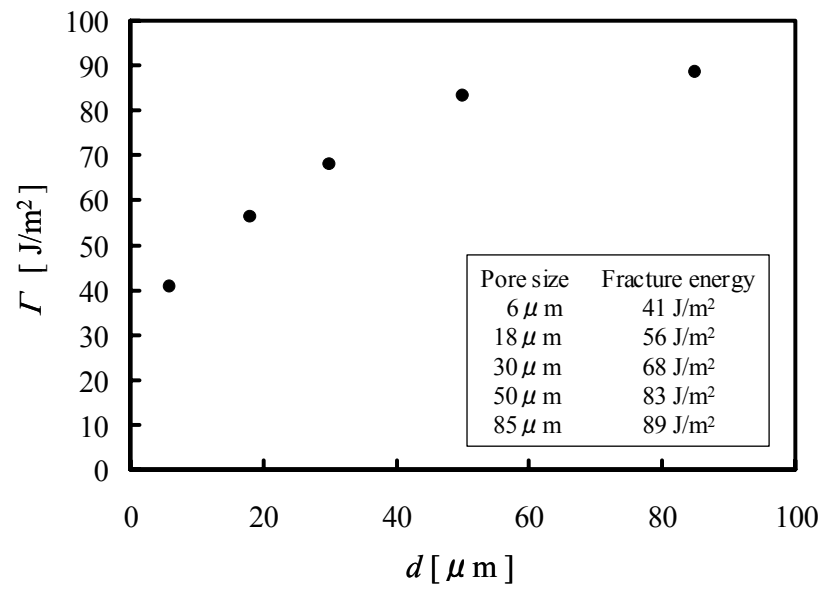

Fig. 8 Fracture energy obtained with the test of Chevron notched specimens.

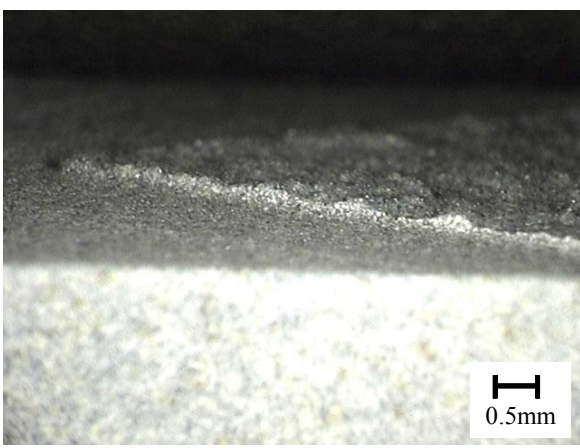

(a)

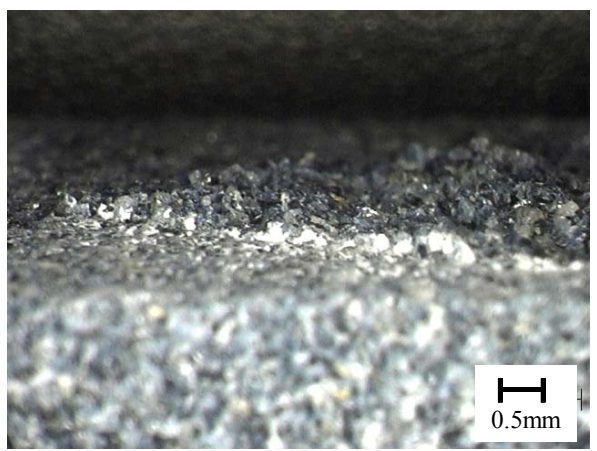

(b)

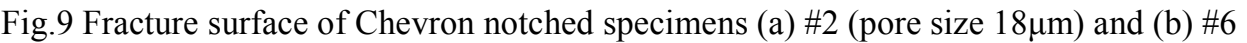
$(85 \mu \mathrm{m})$ observed by CCD camera.

\subsection{Fracture Strength Evaluation of Porous Ceramics}

It has been presented that the fracture strength of porous ceramics are also dominated by the defect size similarly to those of high-density ceramics, and the fracture strength of porous ceramics is derived from the peculiar defect and the pore sizes on the basis of the thought that the fracture occurs when the stress intensity factor at the crack tip reaches the intrinsic fracture toughness of the material (see Fig.3). This result shows that the fracture strength of the material is dominated by a peculiar defect and pores combined with the defect.

The apparent fracture toughness value of the SENB specimen with a small notch depth is lower than that with a relatively large notch. It is thought that the micro-structures at the crack tip play influential part for the fracture in case of a small notch depth. Thus, the process zone fracture model ${ }^{(9)}$ at the crack tip is discussed (Fig.10).

The observations by the laser microscope indicated that unevenness of the fracture surfaces almost corresponds to the sum of the crystal grain size and $2 \mathrm{x}$ average pore size. 2 $\mathrm{x}$ average pore size almost corresponds to the maximum pore size. It is thought that such unevenness observed at the crack tip dominates the fracture of the material.

It is also thought that the fracture of a cracked specimen occurs in the region $s$, which is occupied by grain boundary in the crack propagation direction at the crack tip and pores adjacent to the grain boundary. However, the micro-structures in the region $s$ are complex and it is difficult to specify the origin of the facture and the stress state. Thus, it is assumed 
that the fracture of the material occurs when the average tensile stress in the region $s$ reaches to the intrinsic fracture strength $\sigma^{*}$ of the material.

The tensile stress $\sigma$ at $r$ from the tip of a crack of length $2 a$ is expressed by the following equation when the tensile stress $\sigma_{0}$ perpendicular to the crack applies to infinite plate.

$$
\sigma=\sigma_{0} \frac{a+r}{\sqrt{2 a r+r^{2}}}
$$

It is assumed that the fracture occurs when the average tensile stress $\bar{\sigma}$ in the region $s$ corresponds to the intrinsic strength $\sigma^{*}$ of the material.

$$
\bar{\sigma}=\frac{1}{s} \int_{0}^{s} \sigma d r=\frac{\sigma_{0}}{s} \int_{0}^{s} \frac{a+r}{\sqrt{2 a r+r^{2}}} d r=\frac{\sigma_{0}}{s} \sqrt{2 a s+s^{2}}=\sigma^{*}
$$

The $\sigma_{0}$ corresponds to the fracture strength of the cracked specimen $\sigma_{\mathrm{c}}$, and the equation (8) is rearranged as follows.

$$
\sigma_{\mathrm{c}}=\frac{s}{\sqrt{2 a s+s^{2}}} \sigma^{*}=\frac{1}{\sqrt{1+\frac{2 a}{s}}} \sigma^{*}
$$

The region $s$ is expressed on the basis of the observations of the micro-structures as follows.

$$
s=\delta_{\max }+2 d
$$

Where, $\delta_{\max }$ and $d$ denote the maximum grain size and the average pore size, respectively. Then, the fracture strength of the cracked specimen is derived from the equation (9) as follows.

$$
\sigma_{\mathrm{c}} / \sigma^{*}=\frac{1}{\sqrt{1+\frac{2 a}{\delta_{\max }+2 d}}}
$$

The intrinsic strength $\sigma^{*}$ of the material is regarded as a fracture strength of the plain specimen without a peculiar defect $2 \alpha$. Therefore, the intrinsic strength of the material is obtained from the equation (6) as follows.

$$
\sigma^{*}=\frac{C}{\sqrt{d}}
$$

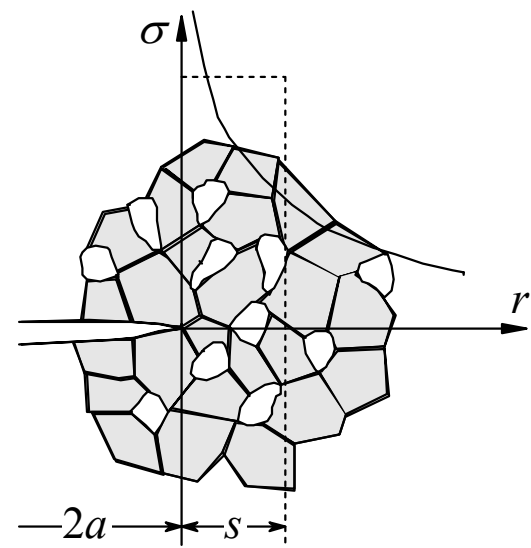

Fig.10 Schematic illustration about process zone of fracture model.

The fracture strength data of the cracked specimen are arranged based on the equation (11) are shown in Fig.11. A curve in this figure is obtained by the equation (11). It is obvious that the fracture strength of the cracked specimen is expressed regardless of the crack and the pore sizes by considering the effects of the crystal grain and the pore at the 
crack tip. It is presented that the intrinsic strength $\sigma^{*}$ of the material and the fracture strength of the cracked specimen can be evaluated if the fracture strength of the plain specimen of porous ceramics is expressed by the equation (6).

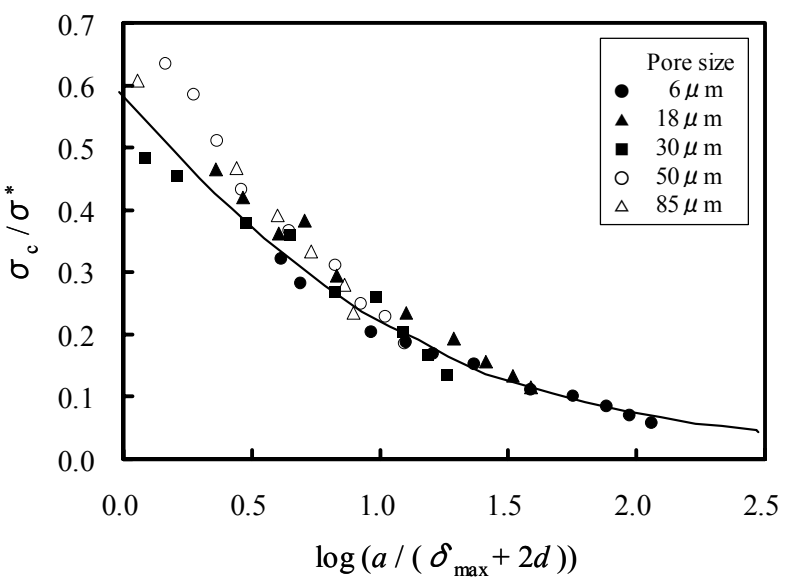

Fig.11 Effect of crack size $a /\left(\delta_{\max }+2 d\right)$ on fracture strength of cracked specimens.

\section{Conclusions}

Fracture strength properties of porous ceramics that have almost equal porosity and significantly different pore sizes were evaluated, and following results were obtained.

(1) Although the fracture strength of the porous ceramic samples tested in this study is lower than those of high-density ceramics, the dispersion of fracture strength data is few. It is thought that the reason for it is random dispersion of many pores which will be origin of the fracture.

(2) Fracture strength of the plain specimen depends on the pore size, and decreases with increase of the pore size. On the fracture surfaces of the specimen, peculiar defects were observed. The relation based on linear fracture mechanics between the defect size and the fracture strength is valid when the one that a pore accompanies with the peculiar defect observed in fracture surface of the material was thought to be a defect size.

(3) Unevenness on the facture surfaces is remarkable for the materials of large pore sizes, and the fracture energy of porous ceramics increases with increase of the pore size. It is deduced that such an increase of the fracture energy is based on the suppression of the crack propagation associated with a crooked propagation path.

(4) By considering the effects of the crystal grain and the pore sizes in the process zone fracture model at the crack tip, the fracture strength of the cracked porous ceramics can be evaluated regardless of the pore size.

\section{References}

(1) Ohji, T., New Performance of Porous Ceramics as Structural Components, National Institute of Advanced Industrial Science and Technology (AIST Today), Vol.1-3 (2001), pp.28-31.

(2) Duckworth W., Discussion of Ryshkewitch Paper by Winston Duckworth, Journal of American Ceramic Society, Vol.36, No.3 (1953), p.68.

(3) Nishikawa, T. et al., Effects of Porosity and Pore Morphology on Mechanical Properties of Porous Alumina, Journal of the Society of Materials Science, Japan, Vol.50, No.6 (2001), pp.625-629.

(4) Sakaida, Y. and Tanaka, K., Evaluation of Fracture Toughness of Porous Ceramics, Transactions of the Japan Society of Mechanical Engineers, Series A, Vol.67, No.660 (2001), pp.116-123. 
(5) Tanaka, K. et al., Bending Strength of Smooth and Notched Specimens of Porous Silicon Carbide, Transactions of the Japan Society of Mechanical Engineers, Series A, Vol.65, No.640 (1999), pp.1-8.

(6) Tanaka, K. et al., Evaluation of Fracture Strength of Porous Ceramics Based on R-Curve Method, Transactions of the Japan Society of Mechanical Engineers, Series A, Vol.69, No.685 (2003), pp.21-28.

(7) Miyata, H. et al., Proposal on the Method of Fixing Proof Strength for Ceramic Components, JSME Int. J. Series I, Vol.35, No.1 (1992), pp.123-131.

(8) Miyata, H. et al., Effect of Micro-Damage on Fracture Strength of Structural Ceramics, Journal of the Japanese Society for Strength and Fracture of Materials, Vol.21, No.2 (1986), pp.45-59.

(9) Takahashi, I. et al., Effect of Size and Notch Root Radius on Fracture Strength of Engineering Ceramics, Yogyo-Kyokai-Shi, Vol.93, No.4 (1985), pp.186-194. 